\title{
Extended Techniques for Transmit Antenna Selection With STBCs
}

\author{
Renato Machado, Student Member, IEEE, and Bartolomeu F. Uchôa-Filho, Member, IEEE
}

\begin{abstract}
In this paper, we consider transmit antenna selection (TAS) with space-time block codes (STBCs). We consider a general framework for TAS which, besides antenna subset selection, includes the possibility of having more than one STBC to choose from as well as non-uniform power allocation. Also, to limit the number of transmit $R F$ chains, we require that the maximum (row) rank of the STBC matrices have an upper limit. Simple analysis indicate that transmitting uncoded data via the transmit antenna with the largest Frobenius norm, which can be viewed as a particular case of TAS with orthogonal STBC (OSTBC), not only provides full diversity as if all the transmit antennas were used but also has larger signal-to-noise ratio as compared to TAS with any standard OSTBC (like the Alamouti code). This suggests that TAS with non-orthogonal STBCs should be considered, in particular by means of exploiting instantaneous coherence within subsets of antennas. A few TAS schemes are proposed and computer simulations are used to demonstrate their superior error performance.
\end{abstract}

Index Terms-Antenna selection, channel feedback, diversity, fading channels, MIMO systems, space-time block codes, wireless communications.

\section{INTRODUCTION}

$\mathbf{I}$ $\mathrm{T}$ is well-known that multiple-input multiple-output (MIMO) wireless communications systems have increased capacity [1]-[3] if the number of receive antennas is at least as large as the number of transmit antennas. On the other hand, practical constraints have resulted in the need of systems employing multiple antennas in the transmitter only. In this case, good performance can be achieved by means of spacetime coding. In particular, Alamouti [4] introduced a very interesting space-time coding technique that achieves the same diversity order with two transmit and one receive antennas as maximal-ratio combining (MRC) with one transmit and two receive antennas, thus transferring the diversity gain from the receiver to the transmitter. Alamouti's scheme called the researchers' attention also because of its simplicity and its low decoding complexity; maximum likelihood decoding is simplified because of the orthogonality imposed on the codewords. This technique has been generalized through the concept of orthogonal designs to an arbitrary number of transmit antennas by Tarokh et al. [5], who coined the name space-time block codes (STBCs). In the subclass of STBCs called orthogonal STBCs (OSTBCs), a code designed for $N_{t}$ transmit and $N_{r}$

Manuscript received August 15, 2006; revised July 16, 2007. This work was supported by the Brazilian National Council for Scientific and Technological Development (CNPq) under grants: 141005/2004-1, 302286/2004-7, and 484391/2006-2.

R. Machado and B. F. Uchôa-Filho are with the Communications Research Group, Department of Electrical Engineering, Federal University of Santa Catarina, Florianópolis, SC, 88040-900, BRAZIL. e-mail: \{machado, uchoa\}@eel.ufsc.br. receive antennas has the desired property of achieving full diversity order: $N_{t} N_{r}$. Non-orthogonal STBCs (NOSTBCs), on the other hand, cannot achieve full diversity but they usually have a greater transmission rate. STBCs are indicated when the channel is known only to the receiver, in which case the total average transmit power is distributed uniformly among the transmit antennas.

When a reliable feedback path is available, the transmitter can use side information to improve the system performance in a way that depends on the amount of feedback available. For instance, when full channel state information (CSI) can be made available to the transmitter, transmit beamforming is the best strategy [12, Sec. 6.1]. In effect, transmit beamforming uses all the transmit antennas and distributes the total average power non-uniformly among them.

Deployment of multiple antennas is normally associated with high cost since multiple antennas usually require an equal number of RF chains (transmit amplifiers, modulators, etc.), which are expensive and power-consuming. To circumvent this problem, a subset of the available antennas can be selected, thereby reducing the number of RF chains. The amount of feedback required in this case is much smaller. Such approach is called transmit antenna selection (TAS). The most wellknown TAS scheme has been proposed by Gore and Paulraj [6]. In [6], an OSTBC is used along with the optimal subset of transmit antennas, which is the one whose associated subchannel matrix has the largest Frobenius norm ${ }^{1}$ This translates to maximizing the instantaneous received signal-to-noise ratio (SNR). Although this scheme applies to any OSTBC, the authors have focused on transmit antenna selection with Alamouti (TAS/A), where the Alamouti code [4] is used along with the optimal pair of antennas. An important result proved in [6] is that TAS with OSTBC provides full diversity order, as if all the antennas were used.

In this paper, we consider a general framework for TAS. It includes the possibility of having more than one code to choose from and it allows for power allocation within the selected subset of antennas. It is then shown that the TAS schemes mentioned above as well as some others can be described in this framework. The transmission of uncoded data via the transmit antenna with the largest Frobenius norm is seen as a particular case of TAS with OSTBC, and a simple argument is used to show that this TAS scheme not only provides full diversity but also has SNR larger than that of

${ }^{1}$ The Frobenius norm of a matrix/vector $\boldsymbol{A}$ is defined as:

$$
\|\boldsymbol{A}\|^{2} \triangleq \sum_{i} \sum_{j}\left|A_{i, j}\right|^{2} .
$$


TAS with any standard OSTBC. This fact and an average SNR analysis stimulate the use of TAS with NOSTBCs, an idea already pursued by the authors in [7]. A few TAS schemes are proposed and computer simulations are used to demonstrate their superior error performance.

The remainder of this paper is organized as follows. In Section II, we present the channel model and briefly review STBCs. In Section III, we describe the general framework for TAS with STBCs and discuss some particular TAS schemes. Following an SNR analysis, we propose a new TAS scheme involving NOSTBCs. In Section IV, we present computer simulation results that corroborate the SNR analysis of the previous section. Finally, in Section V, we draw some conclusions and make some final remarks.

\section{Channel Model And STBCS}

In this section, we describe the channel model and present STBCs and their maximum likelihood (ML) detection.

\section{A. MIMO Channel Model}

We consider the MIMO channel with $N_{t}$ transmit antennas and $N_{r}$ receive antennas. The quasi-static, flat Rayleigh fading channel is assumed, i.e., the channel is assumed constant over a frame and varies randomly from one frame to another. The appropriate channel model under this scenario is the one described by the $N_{r} \times N_{t}$ channel gain matrix

$$
\boldsymbol{H}=\left[\begin{array}{ccc}
h_{1,1} & \cdots & h_{1, N_{t}} \\
\vdots & \ddots & \vdots \\
h_{N_{r}, 1} & \cdots & h_{N_{r}, N_{t}}
\end{array}\right]
$$

where $h_{i, j}$ represents the complex gain from transmit antenna $j$ to receive antenna $i$ and, for $i=1, \ldots, N_{r}$ and $j=1, \ldots, N_{t}$, they are modeled as independent zero mean circularly symmetric complex Gaussian random variables with variance 0.5 per dimension. Let $\boldsymbol{x}$ and $\boldsymbol{y}$ be the transmit and receive vectors, of dimensions $N_{t} \times 1$ and $N_{r} \times 1$, respectively. These are complex vectors but the elements of $\boldsymbol{x}$ are constrained to belong to a certain unit-energy signal constellation. The input-output relationship at a given discrete time instant is given by:

$$
\boldsymbol{y}=\sqrt{\frac{E_{s}}{N_{t}}} \boldsymbol{H} \boldsymbol{x}+\boldsymbol{e}
$$

where $e=\left[e_{1} \cdots e_{N_{r}}\right]^{T}$ is the noise vector whose elements (noise samples) are independent zero mean circularly symmetric complex Gaussian random variables with variance $N_{0} / 2$ per dimension $\left(N_{0} / 2\right.$ is the two-sided noise power spectral density). The superscript $T$ indicates matrix transposition. The factor $1 / \sqrt{N_{t}}$ assures that the available symbol energy $E_{s}$ is shared among the $N_{t}$ transmit antennas. If a sequence $\left\{\boldsymbol{x}_{1}, \ldots, \boldsymbol{x}_{N}\right\}$ of $N$ vectors is transmitted the corresponding input-output relationship is given by:

$$
\boldsymbol{Y}=\sqrt{\frac{E_{s}}{N_{t}}} \boldsymbol{H} \boldsymbol{X}+\boldsymbol{E}
$$

where $\boldsymbol{X}=\left[\boldsymbol{x}_{1}, \ldots, \boldsymbol{x}_{N}\right]$ is the transmit block, $\boldsymbol{Y}=$ $\left[\boldsymbol{y}_{1}, \ldots, \boldsymbol{y}_{N}\right]$ is the receive block, and $\boldsymbol{E}=\left[\boldsymbol{e}_{1}, \ldots, \boldsymbol{e}_{N}\right]$ is the noise block. The noise is also assumed to be white across time, i.e., for receive antennas $n$ and $n^{\prime}$ and at time instants $t$ and $t^{\prime}, E\left[e_{n, t} e_{n^{\prime}, t^{\prime}}^{*}\right]=N_{0} \delta_{n, n^{\prime}} \delta_{t, t^{\prime}}$, where $\delta_{m, n}=0$ if $m \neq n$, and $\delta_{m, n}=1$ if $m=n$ (i.e., the Kronecker delta).

\section{B. OSTBCS}

Let $\left\{s_{k}\right\}_{k=1}^{n_{s}}$ be a set of $n_{s}$ scalar complex symbols representing the data sequence to be transmitted. The symbols are constrained to belong to a unit-energy PAM or QAM constellation $\mathcal{S}$. A STBC can be defined as the image of a mapping

$$
\left\{s_{k}\right\}_{k=1}^{n_{s}} \mapsto \boldsymbol{X}
$$

where $\boldsymbol{X}$ is a matrix of dimension $N_{t} \times N$, as defined above, where $N$ is the length of the space-time codeword. We often refer in a loose way to the STBC as the matrix $\boldsymbol{X}$. The STBC is said to be linear if the elements of the matrix $\boldsymbol{X}$ are linear combinations of the symbols $\left\{s_{k}\right\}_{k=1}^{n_{s}}$ and their complex conjugates $\left\{s_{k}^{*}\right\}_{k=1}^{n_{s}}$. The modulation used for transmission in all antennas is also $\mathcal{S}$. So the elements of $\boldsymbol{X}$ are also required to belong to $\mathcal{S}$. (Unless $\mathcal{S}$ is an algebraically closed set, this additional care must be taken). If $\mathcal{S}$ is real (resp., complex) the STBC is said to be real (resp., complex). Since $n_{s}$ data symbols are transmitted over $N$ time intervals, the transmission rate is equal to $R=n_{s} / N$.

A linear STBC $\boldsymbol{X}$ is orthogonal if, for any signal constellation, the following is true:

$$
\boldsymbol{X} \boldsymbol{X}^{H}=\sum_{k=1}^{n_{s}}\left|s_{k}\right|^{2} \cdot \boldsymbol{I}_{N_{t}}
$$

where $\boldsymbol{I}_{N_{t}}$ is the $N_{t}$-dimensional identity matrix, $\left|s_{k}\right|$ denotes the modulus of the complex number $s_{k}$, and the superscript $H$ stands for hermitian. The simplest example of a rate-one OSTBC is obtained by transmission of a single symbol via a single antenna, i.e., $\boldsymbol{X}_{\boldsymbol{T}}=\left[s_{1}\right]$. The receiver performs maximum-ratio combining. We shall refer to this code as the "trivial" OSTBC, which explains the subscript in $\boldsymbol{X}_{\boldsymbol{T}}=[s]$. The SNR per received symbol for $N_{r}$ receive antennas is:

$$
\gamma_{T}=\gamma_{0}\|\boldsymbol{h}\|^{2}
$$

where $\gamma_{0}=E_{s} / N_{0},\|\cdot\|^{2}$ denotes (squared) Frobenius norm, and $\boldsymbol{h}$ is the single-input multiple-output (SIMO) channel column vector. Since $\boldsymbol{h}$ is Gaussian, $\gamma_{T}$ is a chi-squared random variable with $2 N_{r}$ degrees of freedom. A well-known result is that the diversity order, i.e, the slope (in a log$\log$ scale) of the average error probability curve of a digital modulation over a channel with SNR modeled as a chi-squared random variable with $2 d$ degrees of freedom is equal to $d$ [8]. So, the "trivial" OSTBC offers a diversity order of $N_{r}$.

The most important and referenced OSTBC is the Alamouti code, described by:

$$
\boldsymbol{X}_{\boldsymbol{A}}=\left[\begin{array}{cc}
s_{1} & s_{2}^{*} \\
s_{2} & -s_{1}^{*}
\end{array}\right]
$$

Interpreting (7), at time 1 the data symbols $s_{1}$ and $s_{2}$ are transmitted via antennas 1 and 2 , respectively, and at time 2 the symbols $s_{2}^{*}$ and $-s_{1}^{*}$ are transmitted via antennas 1 and 2 , respectively. Thus, the transmission rate is equal to one. 
The ML detection of STBCs assuming perfect channel knowledge at the receiver is described by

$$
\left\{\hat{s}_{k}\right\}_{k=1}^{n_{s}}=\arg \min _{\left\{s_{k}\right\}_{k=1}^{n_{s}} \in \mathcal{S}^{n_{s}}}\left\|\boldsymbol{Y}-\sqrt{\frac{E_{s}}{N_{t}}} \boldsymbol{H} \boldsymbol{X}\right\|^{2}
$$

The orthogonality property in (5) implies that all OSTBCs have the channel decoupling property (CDP), that is, the joint (block) minimization problem in (8) decouples into $n_{s}$ independent scalar minimization problems by means of a linear processing on $\boldsymbol{Y}$ [5]. For example, equation (3) for the Alamouti code with one receive antenna specializes to:

$$
\left[\begin{array}{ll}
y_{1} & y_{2}
\end{array}\right]=\sqrt{\frac{E_{s}}{2}}\left[\begin{array}{ll}
h_{1} & h_{2}
\end{array}\right]\left[\begin{array}{cc}
s_{1} & s_{2}^{*} \\
s_{2} & -s_{1}^{*}
\end{array}\right]+\left[\begin{array}{ll}
e_{1} & e_{2}
\end{array}\right]
$$

The following linear processing on $\boldsymbol{Y}=\left[\begin{array}{ll}y_{1} & y_{2}\end{array}\right]$ produces the desired decoupling:

$$
\begin{aligned}
\tilde{s}_{1} & =h_{1}^{*} y_{1}-h_{2} y_{2}^{*} \\
& =\left(\left|h_{1}\right|^{2}+\left|h_{2}\right|^{2}\right) s_{1}+h_{1}^{*} e_{1}+h_{2} e_{2}^{*} \\
\tilde{s}_{2} & =h_{2}^{*} y_{1}+h_{1} y_{2}^{*} \\
& =\left(\left|h_{1}\right|^{2}+\left|h_{2}\right|^{2}\right) s_{2}+h_{1} e_{2}^{*}+h_{2}^{*} e_{1}
\end{aligned}
$$

where $\tilde{s}_{1}$ and $\tilde{s}_{2}$ are (decoupled) decision variables for $s_{1}$ and $s_{2}$, respectively. The SNR per symbol for the Alamouti scheme is given by:

$$
\gamma_{A}=\gamma_{0} \frac{\left|h_{1}\right|^{2}+\left|h_{2}\right|^{2}}{2}
$$

For two transmit and $N_{r}$ receive antennas, it can easily be shown that the $\mathrm{SNR}$ is:

$$
\gamma_{A}=\gamma_{0} \frac{\|\boldsymbol{H}\|^{2}}{2}
$$

Note that $\gamma_{A}$ is a chi-squared random variable with $4 N_{r}$ degrees of freedom [8]. So the Alamouti scheme extracts a diversity order of $2 N_{r}$ [4]. A general OSTBC for the MIMO ( $N_{t}$ transmit, $N_{r}$ receive antennas) channel modeled as above has SNR per symbol equal to $\gamma_{0} \frac{\|\boldsymbol{H}\|^{2}}{N_{t}}$, and it provides a diversity order of $N_{t} N_{r}$ [5].

\section{Non-OSTBCs}

Although OSTBCs achieve maximum diversity order, there are some restrictions on the existence of general OSTBCs. For example, rate-one complex OSTBCs for more than two transmit antennas do not exist [5]. On the other hand, it has been shown [9], [10] that by relaxing the orthogonality requirement non-OSTBCs (NOSTBCs) of rates greater or equal to 1 can be obtained at the cost of some diversity loss. A quite simple rate-one complex NOSTBC for three transmit antennas is obtained by extending one of the rows of $\boldsymbol{X}_{\boldsymbol{A}}$. Here are three possibilities:

$$
\begin{gathered}
\boldsymbol{X}_{\boldsymbol{E A 1} \mathbf{1}}=\left[\begin{array}{cc}
s_{1} & s_{2}^{*} \\
s_{1} & s_{2}^{*} \\
s_{2} & -s_{1}^{*}
\end{array}\right], \quad \boldsymbol{X}_{\boldsymbol{E} \boldsymbol{A 2}}=\left[\begin{array}{cc}
s_{1} & s_{2}^{*} \\
s_{2} & -s_{1}^{*} \\
s_{1} & s_{2}^{*}
\end{array}\right], \\
\boldsymbol{X}_{\boldsymbol{E} \boldsymbol{A 3}}=\left[\begin{array}{cc}
s_{1} & s_{2}^{*} \\
s_{2} & -s_{1}^{*} \\
s_{2} & -s_{1}^{*}
\end{array}\right]
\end{gathered}
$$

Note that for the extended Alamouti (EA) code, say $\boldsymbol{X}_{\boldsymbol{E A 3}}$, the input-output relationship for the channel $\boldsymbol{h}=\left[\begin{array}{lll}h_{1} & h_{2} & h_{3}\end{array}\right]$ can be equivalently written in two ways, as follows:

$$
\begin{aligned}
& {\left[\begin{array}{ll}
y_{1} & y_{2}
\end{array}\right]=\sqrt{\frac{E_{s}}{3}}\left[\begin{array}{ll}
h_{1} & h_{2} \\
h_{3}
\end{array}\right]\left[\begin{array}{cc}
s_{1} & s_{2}^{*} \\
s_{2} & -s_{1}^{*} \\
s_{2} & -s_{1}^{*}
\end{array}\right]+\left[\begin{array}{ll}
e_{1} & e_{2}
\end{array}\right]} \\
& =\sqrt{\frac{E_{s}}{2}}\left[\sqrt{\frac{2}{3}} h_{1} \sqrt{\frac{2}{3}}\left(h_{2}+h_{3}\right)\right]\left[\begin{array}{cc}
s_{1} & s_{2}^{*} \\
s_{2} & -s_{1}^{*}
\end{array}\right] \\
& +\left[\begin{array}{ll}
e_{1} & e_{2}
\end{array}\right]
\end{aligned}
$$

That is, $\boldsymbol{X}_{\boldsymbol{E} \boldsymbol{A 3}}$ can be seen as the Alamouti code for the multiple-input single-output (MISO) channel $\boldsymbol{h}=$ $\left[\sqrt{\frac{2}{3}} h_{1} \sqrt{\frac{2}{3}}\left(h_{2}+h_{3}\right)\right]$. So $\boldsymbol{X}_{\boldsymbol{E A 3}}$ (and $\boldsymbol{X}_{\boldsymbol{E A 1}}$ and $\boldsymbol{X}_{\boldsymbol{E A 2} \text { ) }}$ has the CDP and the corresponding SNR per symbol for one receive antenna is given by:

$$
\gamma_{E A 3}=\gamma_{0} \frac{\left|h_{1}\right|^{2}+\left|h_{2}+h_{3}\right|^{2}}{3}
$$

Again, $\gamma_{E A 3}$ in (16) is a chi-squared random variable with 4 degrees of freedom. Thus, for one receive antenna each EA code in (14) alone can offer a diversity order of only $2<$ $N_{t}=3$. Of course, the EA codes are not much interesting in the case that the transmitter has no knowledge about the channel. However, with a little bit of feedback and a clever selection scheme these codes can provide both diversity and SNR gains. We show how in the next section.

\section{TAS WITH STBCS}

In this section we assume that a reliable feedback link is available from the receiver to the transmitter, and that the channel is perfectly known to the receiver. We want to design a communications system employing STBCs under the restrictions of low-cost transmitters and very low rate feedback transmission.

\section{A. Transmitter Cost Considerations}

Deployment of multiple antennas is normally associated with a high cost since multiple antennas usually require an equal number of RF chains which are expensive and powerconsuming. However, if the signal transmitted via a certain antenna is equal to (or, more generally, a linear combination of) the signals transmitted via the other antennas at all times then the number of RF chains can be reduced. Clearly, this situation occurs with the EA codes.

The most effective way to reduce the number of RF chains is to transmit signals via a selected subset of the available antennas. While reducing the transmitter cost, antenna selection can be made optimal in the sense of maximizing the transmission rate or minimizing the error probability [11]. Herein we adopt the latter approach, which amounts to maximizing the instantaneous SNR. Next, we give a description of TAS in a framework that is more general than those found in the literature (see, for instance, [11, Chapter 8] and [12, Chapter 10]). 


\section{B. STBCs with Linear Precoding}

When the CSI is known only to the receiver, and multiple transmit antennas are employed, then space-time coding (which spreads the energy uniformly among the transmit antennas) can be used to achieve diversity gain. However, if we assume a reliable feedback link from the receiver to the transmitter, then the CSI can be made available to the transmitter as well. In this case, the transmitter should be optimized based upon this information. Depending on the amount of feedback available, the transmitter can have either full CSI (i.e., the whole channel gain matrix in (1)) or partial CSI (e.g., some statistical information such as the channel correlation matrix [11], or a few bits indicating, for instance, the ordinal number(s) of the transmit antenna(s) with the largest Frobenius norm(s)); in all cases the side information is to be dispatched to the transmitter on a per frame basis [6].

When full CSI is available to the transmitter, the optimal (in the minimum error probability sense) strategy is known as beamforming [12, Sec. 6.1]. In this paper, we assume that the feedback link sends a few bits to the transmitter in the beginning of each frame, in which case the transmission model should consist of linear precoding, as described in [12, Section 10.3]. With some slight modifications, we arrive at:

$$
\boldsymbol{Y}=\sqrt{\frac{E_{s}}{N_{t}}} \boldsymbol{H} \boldsymbol{W} \boldsymbol{X}+\boldsymbol{E}
$$

that is, the transmitter pre-multiplies the STBC matrix $\boldsymbol{X}$ with a weighting matrix $\boldsymbol{W}$ (of dimension $N_{t} \times N_{t}$ ) which is selected from a finite set $\Omega=\left\{\boldsymbol{W}_{1}, \ldots, \boldsymbol{W}_{K}\right\}$ of size $K$, where $K$ is an integer which is only related to the amount of feedback available and is independent of the number of antennas. Each matrix in $\Omega$ is required to satisfy an power constraint $\operatorname{Tr}\left(\boldsymbol{W} \boldsymbol{W}^{H}\right)=N_{t}$, where $\operatorname{Tr}$ stands for trace.

It should be noted that with the transmission model in (17), given $\boldsymbol{X}$, all the receiver needs to do is choose the most appropriate weighting matrix based on the channel knowledge and send $\left\lceil\log _{2}(K)\right\rceil$ bits (an index in $\{1, \ldots, K\}$ ) to the transmitter. The transmitter in turn transmits according to (17), adopting the weighting matrix from $\Omega$ with this index. When $K$ is very small, precoding takes the particular form of TAS. In this case, the columns of $\boldsymbol{W}$ constitute a subset of the columns of the identity matrix. In the remainder of this paper, we discuss TAS only (as our restrictions are having a small number of RF chains and having a small $K$ ). However, we extend the procedure above to include the possibility of having more than one STBC to choose from and to allow for power allocation within the selected subset of antennas.

\section{General Framework for TAS}

For the remainder of this paper, TAS will be modeled as:

$$
\boldsymbol{Y}=\sqrt{\frac{E_{s}}{n_{t}}} \boldsymbol{H} \boldsymbol{W}_{k} \boldsymbol{X}_{k}+\boldsymbol{E}
$$

where the pair $\left(\boldsymbol{W}_{k}, \boldsymbol{X}_{k}\right)$ must be chosen on a per frame basis from the finite set

$$
\Omega=\left\{\left(\boldsymbol{W}_{1}, \boldsymbol{X}_{1}\right), \ldots,\left(\boldsymbol{W}_{K}, \boldsymbol{X}_{K}\right)\right\}
$$

For each pair $\left(\boldsymbol{W}_{k}, \boldsymbol{X}_{k}\right)$, the weighting matrix $\boldsymbol{W}_{k}$ determines a subset of $n_{t}^{k}\left(n_{t}^{k} \leq N_{t}\right)$ transmit antennas from the $N_{t}$ transmit antennas available for transmission of code $\boldsymbol{X}_{k}$ possibly with non-uniform power allocation. The dimensions of the matrices $\boldsymbol{W}_{k}$ and $\boldsymbol{X}_{k}$ are $N_{t} \times n_{t}^{k}$ and $n_{t}^{k} \times N^{k}$, respectively, where $N^{k}$ is the length of the $k$-th space-time codeword. The average power constraint becomes $\operatorname{Tr}\left(\boldsymbol{W}_{k} \boldsymbol{W}_{k}{ }^{H}\right)=n_{t}^{k} . N_{t}$ is fixed, whereas the parameters $n_{t}^{k}, N^{k}$, and, of course, the matrices elements may vary with $k$. We shall keep the rate constant herein, but this is not a requirement. To limit the transmitter cost, we should make sure that the row rank of $\boldsymbol{X}_{k}$ for all $k$ is upper bounded by some constant representing the number of required RF chains.

\section{Design Examples}

We now present examples of TAS schemes taken from the literature and show how they can be described in the context of the previous section. The expression for the instantaneous SNR for each scheme is also given, where we assume that the MIMO channel matrix $\boldsymbol{H}$ obeys the model described in Section II-A. Let $C_{k}$ be the random variable implied by the Frobenius norm of the $k$-th column of $\boldsymbol{H}$, that is:

$$
C_{k}=\sum_{i=1}^{N_{r}}\left|h_{i, k}\right|^{2}, \quad k=1,2, \cdots, N_{t}
$$

By assumption on $\boldsymbol{H}$, we know that $C_{k}$ are independent and identically distributed chi-squared random variables. We now define a new set of random variables $C_{(k)}$ by rearranging the $C_{k}$ 's in an descending order of magnitude: $\max _{k}\left\{C_{k}\right\}=$ $C_{(1)} \geq C_{(2)} \geq \cdots \geq C_{\left(N_{t}\right)}=\min _{k}\left\{C_{k}\right\} . C_{\left(N_{t}-i+1\right)}$ is called the $i$-th order statistics [13]. Also, we define $C_{(k)+(l)}$ as the random variable implied by the Frobenius norm of the column vector obtained by the (non-coherent) sum of the two columns of $\boldsymbol{H}$ with the $k$-th and the $l$-th largest Frobenius norms. Clearly, $C_{(k)+(l)}=C_{(l)+(k)}$. It is interesting to note that the inequality $C_{(k)+(l)}>C_{(k)}+C_{(l)}$ holds true on occasion, i.e., depending on the channel realization.

Example 1: Consider the TAS strategy that selects on a per frame basis the best antenna from a set of $N_{t}$ transmit antennas for transmission of the "trivial" OSTBC $\boldsymbol{X}_{\boldsymbol{T}}$. A single symbol is sent via the selected antenna and the receiver performs maximum-ratio combining. This scheme was proposed by Chen et al. in [14], [15], wherein it was termed TAS/MRC. For consistency of notation, we shall herein refer to this scheme as TAS/T. The associated matrices are: $\boldsymbol{X}_{k}=[s]$, for all $k$, and

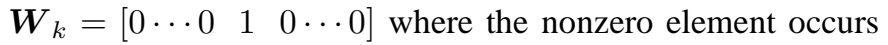
in the $k$-th position. The feedback consists of $\left\lceil\log _{2}\left(N_{t}\right)\right\rceil$ bits. The instantaneous SNR of TAS/T is given by:

$$
\gamma_{T A S / T}=\gamma_{0} C_{(1)}
$$

Example 2: Consider the selection of the best two antennas (out of $N_{t} \geq 2$ transmit antennas) for the transmission of the Alamouti code. The associated matrices are: $\boldsymbol{X}_{k}=\boldsymbol{X}_{\boldsymbol{A}}$, for all $k$, and $\boldsymbol{W}_{k}$, for $k=1, \ldots,\left(\begin{array}{c}N_{t} \\ 2\end{array}\right)$, consist of the matrices of dimension $N_{t} \times 2$ with $\left(N_{t}-2\right)$ all-zero rows and [1 0 ] and $\left[\begin{array}{ll}0 & 1\end{array}\right]$ as the nonzero rows. The selected antennas need 
not be permuted; thus the feedback consists of $\left\lceil\log _{2}\left(\left(\begin{array}{c}N_{t} \\ 2\end{array}\right)\right)\right\rceil$ bits. The instantaneous SNR of TAS/A is given by:

$$
\gamma_{T A S / A}=\gamma_{0} \frac{C_{(1)}+C_{(2)}}{2}
$$

More generally, the instantaneous SNR of TAS with an OSTBC would be:

$$
\gamma_{T A S / O S T B C}=\frac{\gamma_{0}}{n_{t}} \sum_{i=1}^{n_{t}} C_{(i)}
$$

Example 3: Consider the selection of the best three antennas (out of $N_{t} \geq 3$ transmit antennas) for the transmission of the extended Alamouti code $\boldsymbol{X}_{\boldsymbol{E} \boldsymbol{A 3}}$. The associated matrices are: $\boldsymbol{X}_{k}=\boldsymbol{X}_{\boldsymbol{E} \boldsymbol{A 3}}$, for all $k$, and $\boldsymbol{W}_{k}$, for $k=1, \ldots,\left(\begin{array}{c}N_{t} \\ 3\end{array}\right)$, consist of the matrices of dimension $N_{t} \times 3$ with $\left(N_{t}-3\right)$ all-zero rows and $\left[\begin{array}{lll}1 & 0 & 0\end{array}\right],\left[\begin{array}{lll}0 & 1 & 0\end{array}\right]$, and $\left[\begin{array}{lll}0 & 0 & 1\end{array}\right]$ as the nonzero rows. The feedback consists of $\left\lceil\log _{2}\left(\left(\begin{array}{c}N_{t} \\ 3\end{array}\right)\right)\right\rceil$ bits. The instantaneous SNR of TAS/EA3 is given by:

$$
\gamma_{T A S / E A 3}=\gamma_{0} \frac{C_{(1)}+C_{(2)+(3)}}{3}
$$

It should be noted that this selection strategy neglects the fact that the sum of two antennas with small individual Frobenius norms may have norm greater than $C_{(2)+(3)}$. Nevertheless, the simplified selection strategy yields very good results.

Example 4: Consider the selection of either the best two antennas for the transmission of the Alamouti code or the best three antennas (out of $N_{t} \geq 3$ transmit antennas) for the transmission of the extended Alamouti code $\boldsymbol{X}_{\boldsymbol{E} \boldsymbol{A 3}}$. The associated set $\Omega$ is the (disjoint) union of the two sets of matrices pairs in the Examples 2 and 3. This TAS scheme was proposed by the authors in [7] and will be termed herein hybrid-1. Considering antenna permutation in this case amounts to including in $\Omega$ the NOSTBCs $\boldsymbol{X}_{\boldsymbol{E} \boldsymbol{A 1}}$ and $\boldsymbol{X}_{\boldsymbol{E} \boldsymbol{A 2}}$, attached to the same weighting matrices in Example 3. Note that, once the best three antennas are selected, permuting $h_{(2)}$ and $h_{(3)}$ in (16) makes no difference. That is why there are only three essentially different EA codes. With permutations, the hybrid scheme will be called hybrid-1p. The feedback consists of $\left\lceil\log _{2}\left(3\left(\begin{array}{c}N_{t} \\ 3\end{array}\right)+\left(\begin{array}{c}N_{t} \\ 2\end{array}\right)\right)\right\rceil$ bits. The instantaneous SNR of TAS/hybrid-1 and TAS/hybrid-1p are, respectively:

$$
\gamma_{T A S / \text { hybrid-1 }}=\max \left\{\gamma_{T A S / A}, \gamma_{T A S / E A 3}\right\}
$$

and

$$
\begin{aligned}
\gamma_{T A S / \text { hybrid-1p }}= & \max \left\{\gamma_{T A S / A}, \gamma_{T A S / E A 1}\right. \\
& \left.\gamma_{T A S / E A 2}, \gamma_{T A S / E A 3}\right\}
\end{aligned}
$$

\section{E. Average SNR Analysis}

In this section, we present an average SNR analysis of the schemes given in Examples 1-4. As a consequence of the results we propose a new hybrid TAS scheme, which outperforms the previous ones.

Let us begin by defining $S_{n_{t}}$ (for $n_{t}=1, \ldots, N_{t}$ ) as

$$
S_{n_{t}} \triangleq \frac{1}{n_{t}} \sum_{i=1}^{n_{t}} C_{(i)}
$$

So, $S_{1}=C_{(1)}, S_{2}=\frac{C_{(1)}+C_{(2)}}{2}$, and so on. Now note that $S_{n_{t}}$ decreases as $n_{t}$ increases from 1 to $N_{t}$. Therefore, TAS with standard OSTBCs should not be used. That is, if $\Omega$ were to contain OSTBCs only then the "trivial" OSTBC would always be the best selection, not only on the average but for any channel realization. Moreover, TAS with any OSTBC and in particular with the "trivial" OSTBC provides the maximal diversity order, as if all the antennas were used, as proved in [14]. Therefore, by the decreasing property of $S_{n_{t}}$, we can assure that the largest (instantaneous or average) SNR gain is achieved with TAS with the "trivial" OSTBC (among all OSBTCs). This leads us to consider the "trivial" as the only OSTBC, and possibly some NOSTBCs, in designing $\Omega$. The natural hybrid scheme, termed herein hybrid-2, is described as the union of TAS/T and TAS/hybrid-1p. The feedback consists of $\left\lceil\log _{2}\left(3\left(\begin{array}{c}N_{t} \\ 3\end{array}\right)+N_{t}\right)\right\rceil$ bits. The instantaneous SNR is given by:

$$
\begin{aligned}
\gamma_{T A S / \text { hybrid-2 }}= & \max \left\{\gamma_{T A S / T}, \gamma_{T A S / E A 1},\right. \\
& \left.\gamma_{T A S / E A 2}, \gamma_{T A S / E A 3}\right\}
\end{aligned}
$$

The average SNR analysis of TAS schemes would involve the knowledge of the probability density function (PDF) and cumulative distribution function (CDF) of the order statistics, which are in fact well-understood [13]. A closed-form expression for the expected value of $C_{(i)}$ as well as a table with the expected value of $C_{(1)}$ and $C_{(2)}$, for $N_{t}=3, \ldots, 6$ and $N_{r}=2, \ldots, 4$ can be found in [6]. In that paper an exact average SNR analysis of TAS/A has been presented. Chen et al. have derived exact bit error probability expression, for binary phase shift keying (BPSK), of TAS/A in [16], and of TAS/T in [14], [15]. The same analysis for the hybrid TAS schemes would be somewhat more complicated, as the SNR in those cases are expressed as the largest random variable from a set of dependent random variables which are themselves functions of order statistics. We get around this problem by presenting the CDF of the instantaneous SNRs for the TAS schemes in Figures 1 and 2, for (a) $N_{t}=3$, (b) $N_{t}=4$, (c) $N_{t}=5$, and (d) $N_{t}=6$ transmit antennas. In these figures, where we have set $\gamma_{0}=1(0 \mathrm{~dB})$, solid lines represent the CDF of $\gamma_{T A S / h y b r i d-2}$ and dashed lines the CDF of the instantaneous SNR for the other TAS scheme. The average SNRs of the two schemes in each figure appear as vertical lines.

In Figure 1, we present the CDFs of $\gamma_{T A S / h y b r i d-2}$ and of $\gamma_{T A S / A}$. As expected, the average SNR of each scheme gets larger as the number of transmit antennas increases. The average SNR gain of TAS/hybrid-2 over TAS/A decreases as $N_{t}$ increases. It is about $1.51 \mathrm{~dB}, 1.42 \mathrm{~dB}, 1.35 \mathrm{~dB}$, and 1.33 $\mathrm{dB}$ for $N_{t}$ varying from 3 to 6 , respectively. The reason for this behavior is that as $N_{t}$ increases the best norms become more uniform, i.e., $S_{2}$ (see (26)) tends to $S_{1}$ as $N_{t}$ increases. We can also see this decrease in SNR gain as a function of $N_{t}$ from the code selection statistics. In simulating TAS/hybrid-2, it was seen that the "trivial" code is the selected code for over $76 \%$ of the channel realizations when $N_{t}=3$, and $51 \%$ when $N_{t}=6$.

In Figure 2, we present the CDFs of $\gamma_{T A S / h y b r i d-2}$ and of $\gamma_{T A S / T}$. Here the average SNR gain of TAS/hybrid-2 over 

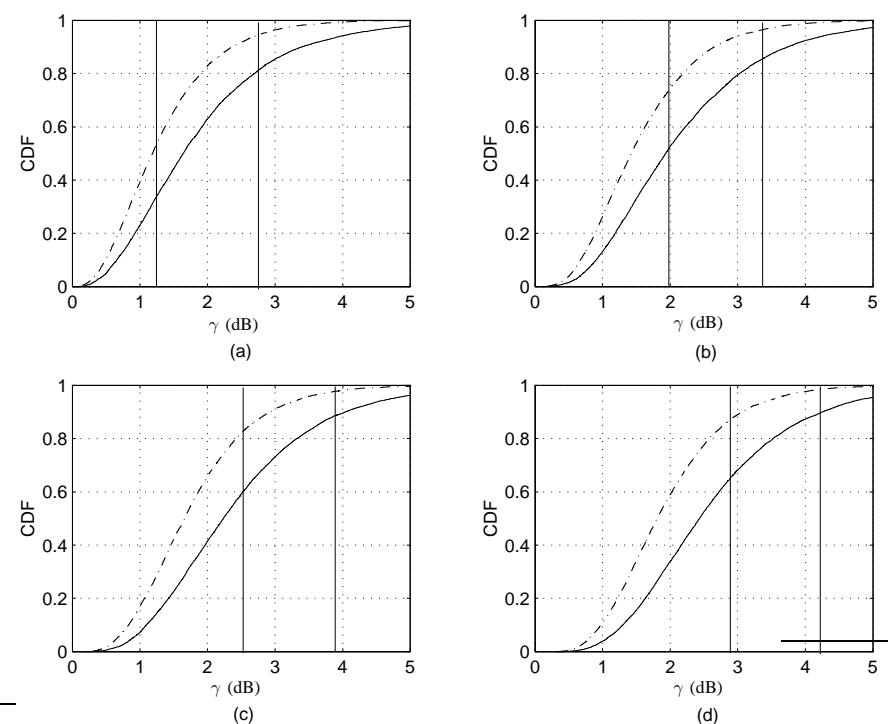

(d)

Fig. 1. CDF of $\gamma_{T A S / h y b r i d-2}$ (solid lines) and $\gamma_{T A S / A}$ (dashed lines) for (a) $N_{t}=3$, (b) $N_{t}=4$, (c) $N_{t}=5$, and (d) $N_{t}=6$ transmit antennas, and $\gamma_{0}=1(0 \mathrm{~dB})$. Vertical lines indicate average SNRs. $N_{r}=1$.

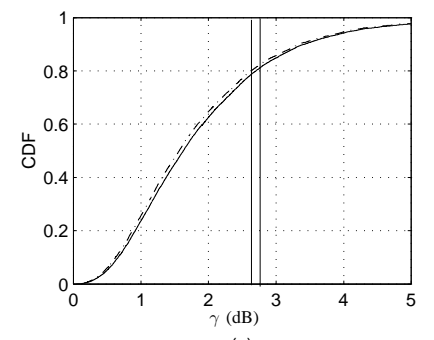

(a)

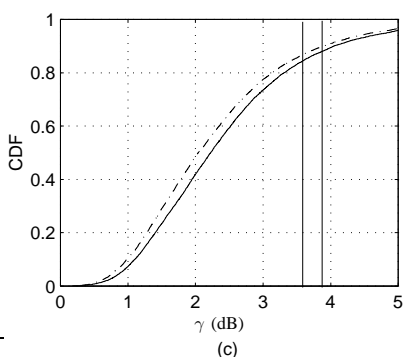

(c)

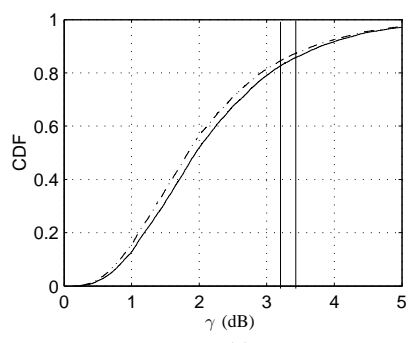

(b)

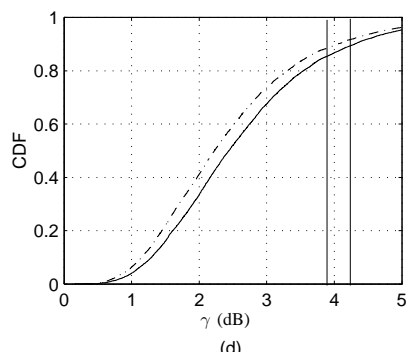

(d)
Fig. 2. CDF of $\gamma_{T A S / h y b r i d-2}$ (solid lines) and $\gamma_{T A S / T}$ (dashed lines) for (a) $N_{t}=3$, (b) $N_{t}=4$, (c) $N_{t}=5$, and (d) $N_{t}=6$ transmit antennas, and $\gamma_{0}=1(0 \mathrm{~dB})$. Vertical lines indicate average SNRs. $N_{r}=1$.

TAS/T increases with $N_{t}$. It is about $0.13 \mathrm{~dB}, 0.23 \mathrm{~dB}, 0.29$ $\mathrm{dB}$, and $0.33 \mathrm{~dB}$ for $N_{t}$ varying from 3 to 6 , respectively. The reason for this is that the "trivial" OSTBC belongs to $\Omega$ in the two TAS schemes. The average SNR advantage accrues from the opportunistic selection of the EA codes. Although the gains in terms of average SNR are small, the gains as measured by the SNR required to achieve a given bit error rate (BER) are more significant, as we shall see next.

\section{Simulation Results}

In this section, we compare the TAS schemes discussed in Section III in terms of their error performance, by means of computer simulations. We present the BER for BPSK

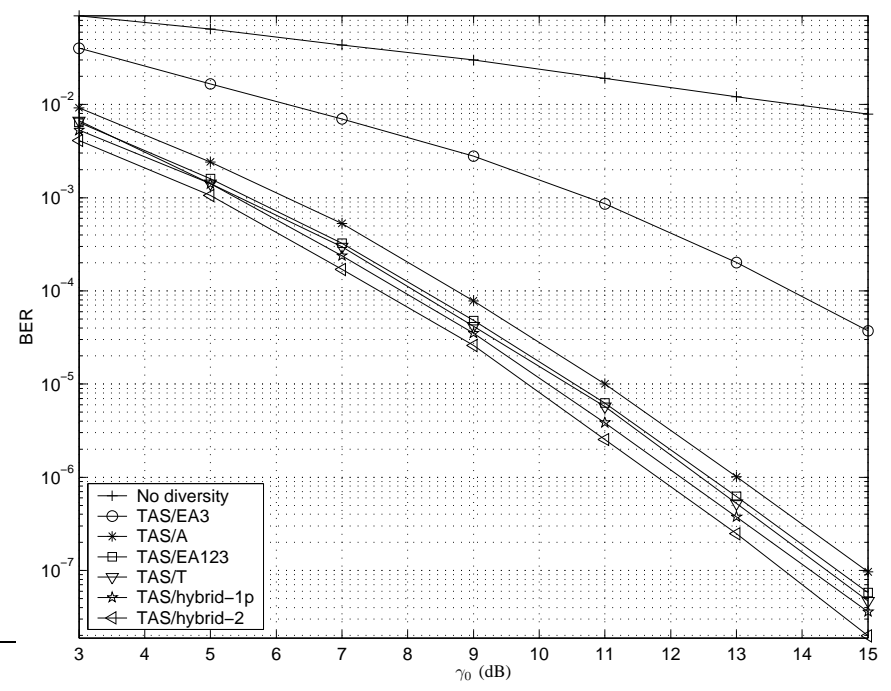

Fig. 3. BER of TAS/EA3, TAS/A, TAS/EA123, TAS/T, TAS/hybrid-1p, and TAS/hybrid-2, for BPSK, $N_{t}=6$, and $N_{r}=1$.

under flat, quasi-static fading. In all simulations we have assumed $N_{r}=1$ receive antenna, 130 symbols per frame, and considered as stopping criterion the occurrence of 10,000 symbol errors per each SNR. The maximum number of RF chains for all schemes simulated is 2 . The BER for the case of no diversity $\left(N_{t}=N_{r}=1\right)$ is also plotted to serve as a reference.

Figure 3 shows, for $N_{t}=6$ transmit antennas, the BER of TAS/EA3, TAS/A, TAS/EA123, TAS/T, TAS/hybrid-1p, and TAS/hybrid-2. The scheme TAS/EA123, not previously described, corresponds to the selection of the best three antennas and the best EA code (EA1, EA2 or EA3). It is just the scheme TAS/hybrid-2 without the "trivial" code. From the simulation results, we observe that TAS/EA3 does not achieve full (i.e., order 6) diversity. This would be expected since EA3 is a NOSTBC. However, it is interesting to note that when permutation of the selected antennas is considered (TAS/EA123), full diversity can be extract despite the fact that in this case all codes in $\Omega$ are non-orthogonal.

The contribution of the EA codes to the overall gain obtained by TAS/hybrid- 2 can be quantified by the gain of TAS/EA123 over TAS/A in Figure 3, which is $0.5 \mathrm{~dB}$. About the same gain is achieved by TAS/T over TAS/A. The complete system (i.e., TAS/hybrid-2), as can be seen from Figure 3, shows an improvement of about $1.3 \mathrm{~dB}$ over TAS/A. This hints that multi-code selection can be interesting.

The number of feedback bits required by TAS/hybrid- 2 for $N_{t}=3,4$, and 5 is, respectively, 3,4 , and 6 . For $N_{t}=6$, there are 66 pairs $\left(\boldsymbol{W}_{k}, \boldsymbol{X}_{k}\right)$ in $\Omega$. With negligible loss of performance, we can discard two such pairs rounding the size of $\Omega$ so that the system requires 6 bits of feedback. These figures represent no more than two extra bits as compared to TAS/A.

Regarding the other curves in Fig. 3, the superior performance of the hybrid schemes corroborates the average SNR advantage of Section III-E. Note that the SNR gain (from the BER curves) of TAS/hybrid-2 over TAS/T is about $0.7 \mathrm{~dB}$ in 


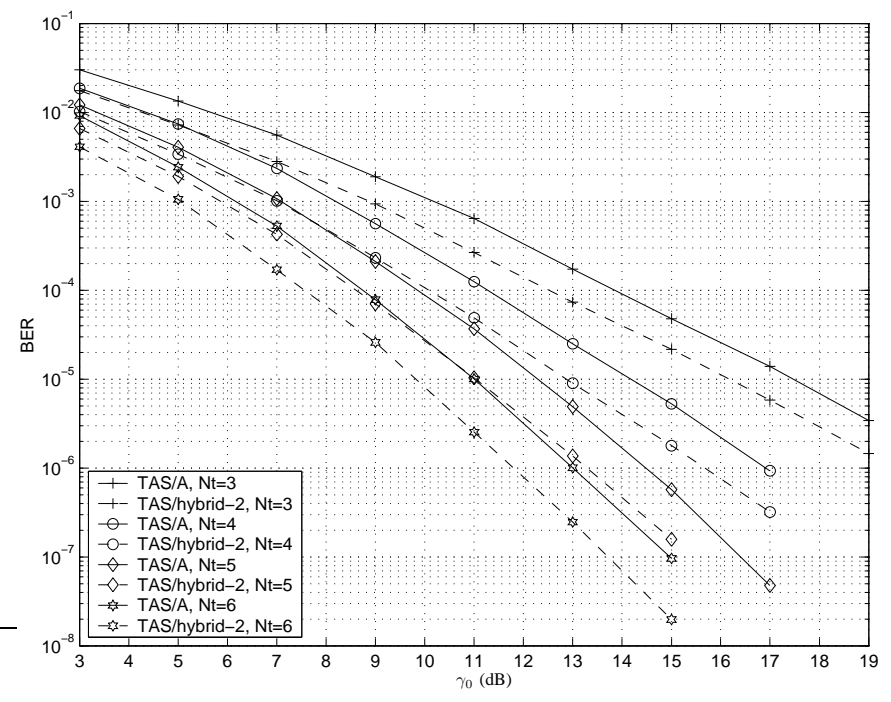

Fig. 4. BER of TAS/hybrid-2 ( $n_{t}=1$ or 3$)$ and TAS/A $\left(n_{t}=2\right)$ for BPSK, $N_{t}=3, \ldots, 6$, and $N_{r}=1$. Full (i.e., order $N_{t}$ ) diversity is achieved as if all the transmit antennas were used.

the high-SNR regime, while the average SNR gain found in Section III-E was only $0.33 \mathrm{~dB}$.

In order to demonstrate the full diversity gain obtained with TAS, even in the presence of NOSTBCs, the BER of TAS/hybrid-2 and TAS/A is shown in Figure 4. Note that the slope of the curves is related to $N_{t}$.

\section{CONClusions AND FinAl REMARKS}

In this paper we have considered a general framework for transmit antenna selection with space-time block codes. In general, transmit antenna selection schemes require very little feedback as compared with optimal transmit beamforming. Furthermore, the nature of the transmit antenna selection approach is such that the amount of feedback is independent of the number of antennas. Another important advantage of transmit antenna selection is the reduced transmitter cost as fewer RF chains are required.

In our general framework, based on the instantaneous channel conditions, the transmitter chooses a STBC and a weighting matrix for transmission aiming at minimizing the error probability. It was seen that the "trivial" orthogonal STBC - obtained by transmitting uncoded data via a single antenna - is the best choice within the class of orthogonal STBCs, both in terms of instantaneous (and average) SNR and in terms of the number of transmit RF chains. It was then shown that to improve error performance further nonorthogonal STBCs have to be included as options for selection. A few transmit antenna selection schemes containing simple non-orthogonal STBCs as well as the "trivial" orthogonal STBC were proposed, and their error performance was evaluated through computer simulations.

Perhaps the most important conclusion that can be drawn from the results in this paper is that in transmit antenna selection non-orthogonal STBCs should be exploited. The schemes proposed in Sec. III-D are only simple special cases, that may serve as an indication of the improved performance obtained in this way. Another interesting scheme wherein non-orthogonal STBCs are considered has been proposed by Akhtar and Gesbert [17], [18]. The so-called Group-Coherent Codes (GCCs) are non-orthogonal STBCs that are used in a selection scheme based upon the instantaneous channel conditions. They can be described in the context of the framework in Sec. III-C, and correspond to no-antenna, but purely code selection, i.e., the $n_{t}=N_{t}$ case. For example, the simplest GCC would be described as $\boldsymbol{X}_{1}=\boldsymbol{X}_{2}=\left[\begin{array}{ll}\boldsymbol{X}_{\boldsymbol{A}}^{T} & \boldsymbol{X}_{\boldsymbol{A}}^{T}\end{array}\right]^{T}$, and $\boldsymbol{W}_{k}=\operatorname{diag}\left(\boldsymbol{I}_{2},(-1)^{k+1} \boldsymbol{I}_{2}\right)$, for $k=1,2$, with $N_{t}=$ $n_{t}=4$. It requires 1-bit feedback. Since $\boldsymbol{X}_{k}$ has rank 2 this system requires only two RF chains. If more transmit antennas were available, then the GCCs could be used in combination with antenna selection, thus following the idea of TAS with non-orthogonal STBCs.

Another point that is worth mentioning is the benefits of non-uniform power allocation. If the weighting matrices associated with the NOSTBCs in the framework of Sec. III-C are prepared to distribute the transmit power among the transmit antennas in an optimal fashion, then further coding/array gains can be obtained. Non-uniform power allocation also plays an important role in the case of feedback errors. In this regard, a very interesting result has been presented by Ganesan et al. [19]. According to their analysis, TAS/T experiences diversity loss in the presence of errors in the feedback path. Under these non-ideal conditions, it turns out that TAS/OSTBC with optimal non-uniform power allocation represents a more robust solution. The analysis of the error performance of TAS schemes involving non-orthogonal STBCs for unreliable feedback lies beyond the realm of this paper, but it is an interesting problem for future investigation.

\section{ACKNOWLEDGMENT}

The authors would like to thank Prof. Richard Demo Souza for helpful discussions and for his many suggestions that improved the paper.

\section{REFERENCES}

[1] G. J. Foschini and M. J. Gains, "On limits of wireless communications in a fading environment when using multiple antennas," Wireless Personal Commun. , vol. 6, pp. 311-335, 1998.

[2] I. E. Telatar, "Capacity of multi-antenna Gaussian channels," European Trans. Telecom., vol. 10, pp. 585-595, 1999.

[3] J. H. Winters, "On the capacity of radio communication systems with diversity in Rayleigh fading environments," IEEE Journal on Select Areas in Commun., vol. 5, pp. 871-878, Jun. 1987.

[4] S. M. Alamouti, "A simple transmit diversity technique for wireless communications," IEEE Journal on Selected Areas in Commun., vol. 16, no. 8, pp. 1451-1458, Oct. 1998.

[5] V. Tarokh, Hamid. Jafarkhani, and A. R. Calderbank, "Space-time codes from orthogonal designs," IEEE Trans. on Inform. Theory, vol. 45, no. 5, pp. 1456-1467, Jul. 1999.

[6] D. Gore and A. Paulraj, "MIMO antenna subset selection with spacetime coding," IEEE Trans. on Signal Proc., vol. 50, no. 10, pp. 25802588, Dec. 2002.

[7] R. Machado and B. F. Uchôa-Filho, "Space-time block coding with hybrid transmit antenna/code selection," Proc. of the 2004 IEEE Int. Conf. on Commun. (ICC'04), in CD-ROM, Jun. 2004.

[8] J. G. Proakis, Digital Communications, New York: McGraw Hill, 3rd. edition, 1995.

[9] H. Jafarkhani, "A quasi-orthogonal space-time block code," IEEE Trans. on Commun., vol. 49, no. 1, pp. 1-4, Jan. 2001.

[10] M. Uysal and C. N. Georghiades, "Non-orthogonal space-time block codes for 3Tx antennas," Electronics Letters, vol. 38, no. 25 pp. 16891691, Dec. 2002. 
[11] A. Paulraj, R. Nabar, and D. Gore Introduction to Space-Time Wireless Communications, Cambridge University Press, Cambridge, UK, 2003.

[12] E. G. Larsson and P. Stoica, Space-Time Block Coding for Wireless Communications, Cambridge University Press, Cambridge, UK, 2003.

[13] H. A. David and H. N. Nagaraja, Order Statistics, Third Edition, Wiley Series in Probability and Statistics, John Wiley \& Sons, Inc., 2003.

[14] Z. Chen, B. Vucetic, J. Yuan and K. L. Lo, "Analysis of transmit antenna selection/maximal-ratio combining in rayleigh fading channels," Proceedings of ICCT2003, pp. 1532-1536, 2003.

[15] Z. Chen, B. Vucetic, J. Yuan and K. L. Lo, "Analysis of transmit antenna selection/maximal-ratio combining in rayleigh fading channels," Proc. of the 2003 IEEE Int. Symp. on Inform. Theory (ISIT'03), pp. 94, June-July 2003.

[16] Z. Chen, B. Vucetic, J. Yuan and Z. Zhou, "Performance analysis of the Alamouti scheme with transmit antenna selection in rayleigh fading channels," Proc. of the 2004 IEEE Int. Conf. on Commun. (ICC'04), in CD-ROM, Jun. 2004.

[17] J. Akhtar and D. Gesbert, "Partial feedback based orthogonal block coding," in Proc. of the 2003 IEEE Vehicular Technology Conf. (VTC'03Spring), vol. 1, pp. 287-291, 2003.

[18] J. Akhtar and D. Gesbert, "Extending orthogonal block codes with partial feedback," IEEE Trans. on Wireless Commun., vol. 3, pp. 1959 - 1962, Nov. 2004.

[19] G. Ganesan, P. Stoica, and E. Larsson, "Orthogonal space-time block codes with feedback," Wireless Personal Commun., vol. 28, pp. 287-312, 2004.

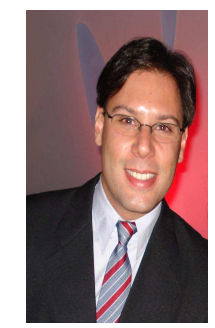

Renato Machado was born in Jaú, SP, Brazil, in 1979. He received the B.S. degree in Electrical Engineering from the São Paulo State University (UNESP), Ilha Solteira, SP, Brazil, in 2001. He received the M.Sc. degree from the Federal University of Santa Catarina (UFSC), Florianópolis, SC, Brazil, in 2004. He was a Visiting Research in the Department of Electrical Engineering, Arizona State University (ASU), Tempe, AZ, U.S.A., from August 2006 to June 2007. He is currently concluding his Ph.D. UFSC, Florianópolis, SC, Brazil. at the Communications Research Group (GPqCom),

Renato Machado is a student member of the Institute of Electrical and Electronics Engineers (IEEE) and the Brazilian Telecommunication Society (SBrT). His research interests include MIMO systems, space-time coding, linear dispersion codes, cooperative diversity, and wireless communications in general.

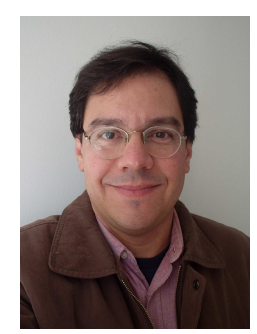

Bartolomeu F. Uchôa-Filho (S'94-M'96) was born in Recife, Brazil, in 1965. He received the B.S.E.E. degree from the Federal University of Pernambuco (UFPE), Recife, Brazil, in 1989; the M.S.E.E. degree from the State University of Campinas (UNICAMP), Campinas, Brazil, in 1992; and the Ph.D. degree in electrical engineering from the University of Notre Dame, Notre Dame, Indiana, U.S.A., in 1996. During 1997-1999 he held a Post-Doctoral position at the State University of Campinas. From August 1999 to January 2000 he was a Visiting Researcher in the Department of Electrical Engineering, Federal University of Santa Catarina, Florianópolis, Brazil. Since February 2000 he has been an Associate Professor in the same Department. His research interests are in the area of coding and information theory, with applications to digital communications systems.

Dr. Uchôa-Filho is a member of Eta Kappa $\mathrm{Nu}$, the IEEE Information Theory Society, the IEEE Communications Society, and the Brazilian Telecommunications Society. For the 2003-2004 period, he served as the Editor-in-Chief of the Journal of the Brazilian Telecommunications Society. 